\title{
The bioluminescent Listeria monocytogenes strain Xen32 is defective in flagella expression and highly attenuated in orally infected BALB/cJ mice
}

\author{
Silke Bergmann ${ }^{1}$, Manfred Rohde $^{2}$, Klaus Schughart ${ }^{1,3}$ and Andreas Lengeling ${ }^{4^{*}}$
}

\begin{abstract}
Background: In vivo bioluminescence imaging (BLI) is a powerful method for the analysis of host-pathogen interactions in small animal models. The commercially available bioluminescent Listeria monocytogenes strain Xen32 is commonly used to analyse immune functions in knockout mice and pathomechanisms of listeriosis.

Findings: To analyse and image listerial dissemination after oral infection we have generated a murinised Xen32 strain (Xen32-mur) which expresses a previously described mouse-adapted internalin A. This strain was used alongside the Xen32 wild type strain and the bioluminescent L. monocytogenes strains EGDe-lux and murinised EGDe-mur-lux to characterise bacterial dissemination in orally inoculated BALB/CJ mice. After four days of infection, Xen32 and Xen32-mur infected mice displayed consistently higher rates of bioluminescence compared to EGDe-lux and EGDe-mur-lux infected animals. However, surprisingly both Xen32 strains showed attenuated virulence in orally infected BALB/c mice that correlated with lower bacterial burden in internal organs at day 5 post infection, smaller losses in body weights and increased survival compared to EGDe-lux or EGDe-mur-lux inoculated animals. The Xen32 strain was made bioluminescent by integration of a lux-kan transposon cassette into the listerial flaA locus. We show here that this integration results in Xen32 in a flaA frameshift mutation which makes this strain flagella deficient.
\end{abstract}

Conclusions: The bioluminescent L. monocytogenes strain Xen32 is deficient in flagella expression and highly attenuated in orally infected BALB/c mice. As this listerial strain has been used in many BLI studies of murine listeriosis, it is important that the scientific community is aware of its reduced virulence in vivo.

Keywords: Listeriosis, Flagella, Mouse infection model, Bioluminescent imaging

\section{Findings \\ Background}

Bioluminescent in vivo imaging (BLI) of Listeria monocytogenes infections in mice has generated several new insights into the pathogenesis of listeriosis. For example, the now commercially available Listeria monocytogenes strain Xen32 was first used to demonstrate that the gallbladder is an important organ reservoir of listerial replication and pathogen shedding [1-3]. Since then the Xen32 listerial strain has been used in multiple studies as a tool to study Listeria directed immune mechanisms in knockout mice [4] and kinetics of L. monocytogenes dissemination to target organs of listeriosis such as the

\footnotetext{
* Correspondence: andreas.lengeling@roslin.ed.ac.uk

${ }^{4}$ Infection and Immunity Division, The Roslin Institute and R(D)SVS, University of Edinburgh, Easter Bush Veterinary Campus, Edinburgh EH25 9RG, UK Full list of author information is available at the end of the article
}

bone marrow [5]. More recently, the bioluminescent Xen32 strain has also been used to study transplacental transmission of L. monocytogenes in fetal listeriosis [6,7].

The aim of this study was to use the bioluminescent $L$. monocytogenes strain Xen32 in an oral mouse listeriosis model to analyse the dissemination of the pathogen from the intestine to internal organs. To enable efficient transmission of this listerial strain through the murine gut mucosa we used our previous approach of murinisation to optimise the binding of the listerial surface protein internalin A (InlA) to the murine E-cadherin host receptor [8].

\section{Material and methods}

For oral inoculation of female, 9-10 weeks old BALB/cJ mice (Harlan Winkelmann, Borchen, Germany) we used our previously published mouse infection model [9].

\section{Biomed Central}


All experiments were conducted according to German animal welfare regulations after approval from the Niedersächsisches Landesamt für Verbraucherschutz und Lebensmittelsicherheit (LAVES) as the local authority. The Listeria monocytogenes strains EGDe-lux (LmoEGDe-lux) and L. monocytogenes EGDe-InlA-mur-lux (Lmo-EGDe-mur-lux) have been described previously [10]. L. monocytogenes Xen32 was purchased from Perkin Elmer (Rodgau, Germany) and genetically modified for expression of a mouse-adapted InlA as previously described [8]. Analysis of bacterial organ counts (colony forming units, CFU) was performed as described in Bergmann et al. [9]. BLI images were obtained using an IVIS 200 imaging system (CaliperLS) with integration time of $3 \mathrm{~min}$ (Xen32 strains) or $4 \mathrm{~min}$ (EGDe strains) at a binning of 8 and F/stop of 1. Photon flux was quantified by using the Living Image 3.1 software (CaliperLS). To assess general growth characteristics of the different L. monocytogenes strains, growth curves were performed as previously described [11]. Luminescence was measured by quantifying photon flux of $0.5 \mathrm{ml}$ culture samples from a $50 \mathrm{ml}$ logarithmic L. monocytogenes culture at indicated timepoints on the IVIS 200 imaging system (5 sec integration time, binning of 8 and F/stop of 1). Genomic flaA fragments from L. monocytogenes Xen32 were amplified with flaA forward primer (5'-AGAG AAGTCTTTTCTAAACCGAATGTAGGA-3') and flaA reverse primer $\left(5^{\prime}\right.$-CTAAGGGTAAACAATGTTCGAT AAATG-3'), sequenced and analysed with MacVector 11.0.2 (MacVector Inc., Cambridge, UK). For analysis of flagella expression, listerial strains were grown overnight in $\mathrm{BHI}$ medium at $24^{\circ} \mathrm{C}$ and negatively stained with $2 \%$ uranyl acetate and examined in a Zeiss TEM910 at $80 \mathrm{kV}$. Cell invasion assays with the human colorectal epithelial cell line Caco-is deficient in flagella expression 2 (ATCC HTB-37) and the murine colon carcinoma cell line CT26 (ATCC CRL-2639) were performed as previously described [8]. Statistical analysis of CFU data was performed using the Mann-Whitney $U$ non-parametic test and the GraphPad Prism 5 (version 5.01) analysis software (GraphPad Software Inc.). Survival curves were statistically evaluated by Kaplan-Meier and Log- rank (Mantel-Cox) analyses.

\section{Results and discussion}

The L. monocytogenes strain Xen32 was originally generated by screening a lux-kan transposon integration library of the parental strain $L$. monocytogenes strain 10403S for high levels of bioluminescence [1]. Xen32, also named clone $2 \mathrm{C}$, was selected after in vivo evaluation of strong photon emission in infected BALB/C mice [1]. To further optimise this strain for oral infection challenge in mice we generated an isogenic mutant by replacing the wild type inlA with inlA ${ }^{S 192 N-Y 369 S}$ as previously described [8]. The new murinised $L$. monocytogenes strain was called Xen32-mur and the introduced mutations in the inlA locus validated by sequencing and Western blot analysis of InlA and internalin B protein expression (data not shown). All $L$. monocytogenes strains were profiled for in vitro growth in BHI media and emitted levels of luminescence. While no differences in in vitro growth rates were found between the listerial strains, both Xen32 strains showed higher levels of bioluminescence compared to the EGDe strains (Additional file 1: Figure S1). BALB/cJ mice were intragastrically infected with $10^{10} \mathrm{CFU}$ of $L$. monocytogenes (Lmo) Xen32, Lmo-Xen32-mur, LmoEGDe-lux, or Lmo-EGDe-mur-lux and analysed by BLI for a time period of 9 days post infection (d.p.i.). After 4 days of infection, Lmo-Xen32 and Lmo-Xen32-mur infected mice displayed high levels of light emission in abdominal regions (Figure $1 \mathrm{~A}$ and Additional file 2: Figure S2). The measured level of bioluminescence in these regions was about 10-fold higher in Lmo-Xen32 infected mice compared to Lmo-EGDe-lux infected mice and 5-fold more intense in Lmo-Xen32-mur infected animals compared to Lmo-EGDe-mur-lux infected animals. The intense BLI signals in Lmo-Xen32 and Lmo-Xen32-mur infected mice remained high for one additional day (5 d.p.i.) and decreased then until no further signals remain detectable at 9 d.p.i. (Additional File 2: Figure S2). Surprisingly despite these high levels of bioluminescence, Lmo-Xen32 and Lmo-Xen32-mur infected mice lost less body weight (Figure 1B) and had significantly lower mortality rates compared to LmoEGDe-lux and EGDe-mur-lux infected mice (Figure 1C). In contrast, infection with both EGDe listerial strains resulted in more drastic losses in body weights and reduced survival after 5 d.p.i. (Figure 1C). To further analyse differences in strain virulence we determined bacterial organ loads after 3 and 5 d.p.i. with $5 \times$ $10^{9}$ CFU bacteria. At 5 d.p.i., Lmo-EGDe-lux infected mice had about 10-fold higher CFU loads in mesenteric lymph nodes and 100-fold higher bacterial organ loads in the liver compared to Lmo-Xen32 infected mice (Figure 2B). Higher CFU loads in Lmo-EGDe-mur-lux infected mice were also detected at 3 d.p.i. in the liver compared to Lmo-Xen32-mur infected mice (Figure 2C). These became more prominent at 5 d.p.i. with about 10 -fold higher CFU counts in the intestine and about 100-fold and 1000-fold higher organ loads in spleen and liver, respectively, in Lmo-EGDe-mur-lux infected mice compared to Lmo-Xen32-mur infected animals (Figure 2D). Thus, the higher mortality observed in Lmo-EGDe-lux and Lmo-EGDe-mur-lux infected mice correlated with increased organ loads compared to Lmo-Xen32 and Lmo-Xen32-mur infected mice and not with the level of detected bioluminescence. The 


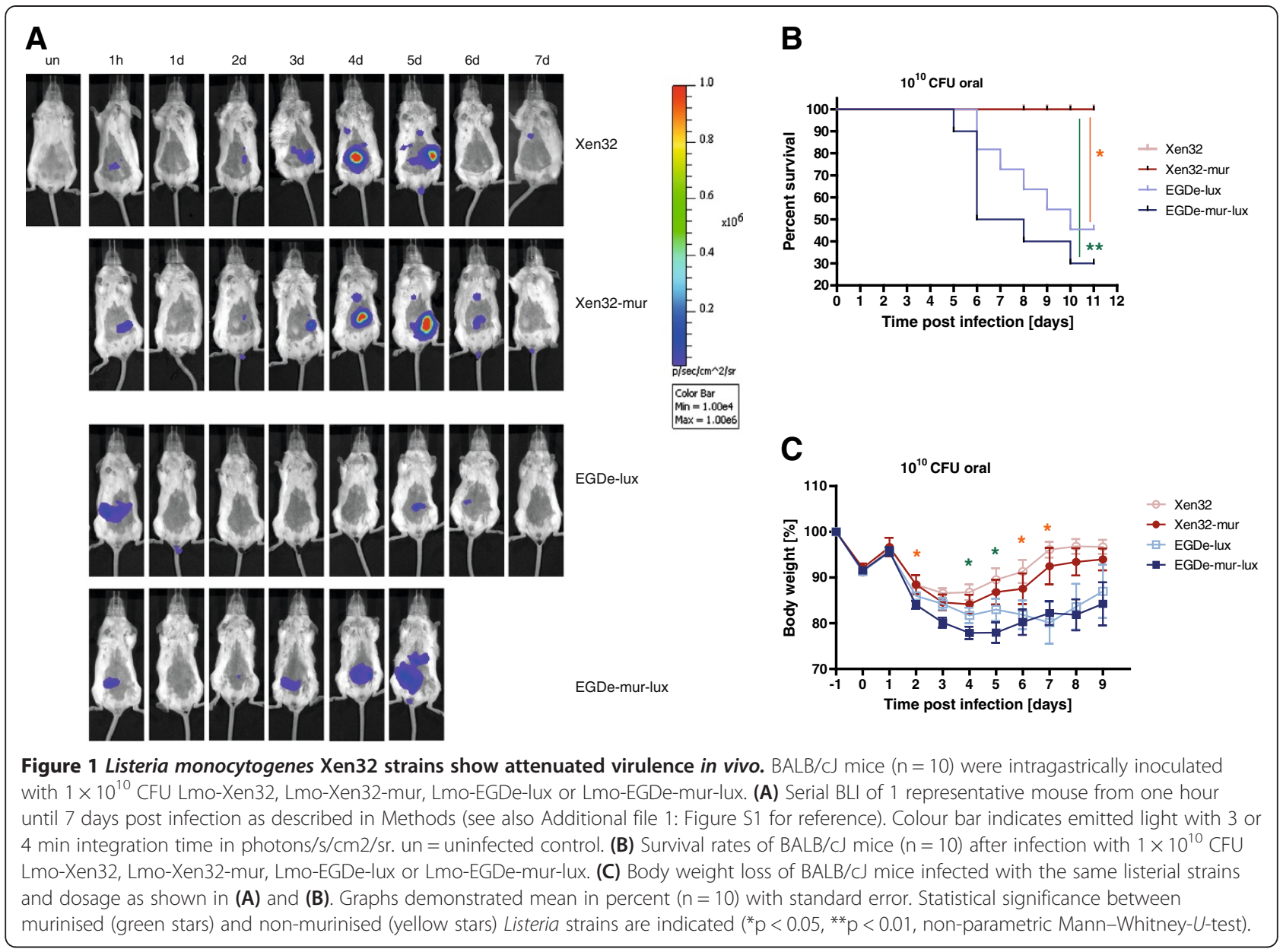

Lmo-Xen32 strains appeared to emit in vivo higher light levels compared to the EGDe listerial strains. However, this was not related to virulence in our mouse infection model. The $\mathrm{LD}_{50}$ for Lmo-Xen32 in BALB/c mice has been described to be four times higher compared to the parental L. monocytogenes strain 10403S [1]. Because the parental $L$. monocytogenes strains $10403 \mathrm{~S}$ and EGDe are known to have similar $\mathrm{LD}_{50}$ dosages in $\mathrm{BALB} / \mathrm{c}$ mice [12-14] we were surprised by the big difference in listeriosis outcome that we observed in this study between mice that were infected with derivates of the 10403S and EGDe strains. In Xen32 the lux operon was integrated via transposon insertion into the flagellin encoding flaA locus [1]. The consequences of this integration have so far not been analysed. Therefore, we sequenced flaA in Lmo-Xen32 and found that the insertion of the lux-kan transposon cassette caused a frameshift mutation which generates an amber stop codon (TAG) 80 bp downstream of the methionin start codon (Additional file 3: Figure S3). This mutation would result in the expression of a truncated, nonfunctional flagellin fusion protein of 27 amino acids instead of the full-length flagellin protein of 287 amino acids. Indeed, when we examined the parental listerial strains Lmo-10403S, Lmo-EGDe, and the bioluminescent strains Lmo-EGDe-lux and EGDe-mur-lux with transmission electron microscopy we found that all these strains clearly expressed peritrichous flagella. In contrast, both Xen32 strains were found to be completely flagelladeficient (Figure 3A). To assess whether the Xen32 mutation influence invasion into human Caco-2 and murine CT26 cells, we performed Gentamicin-protection-invasion assays with Lmo-Xen32, Lmo-Xen32-mur, LmoEGDe-lux and Lmo-EGDe-mur-lux. Lmo-EGDe-mur-lux invaded murine CT26 cells and to a lesser extent also human Caco-2 cells more efficiently than Lmo-EGDe-lux (Additional file 4: Figure S4A, S4C) in line with previous results $[8,10]$. No such differences were detectable between the Lmo-Xen32 and Lmo-Xen32-mur strains (Additional file 4: Figure S4B, S4D) suggesting that flagella are required for optimal invasion of CT26 and Caco- 2 cells as previously demonstrated by O'Neil and Marquis [15].

The possible roles of flagella in listeriosis pathogenesis are diverse. They include host cell adhesion and invasion, injection of virulence factors and initiation and modulation of host inflammation through recognition of 

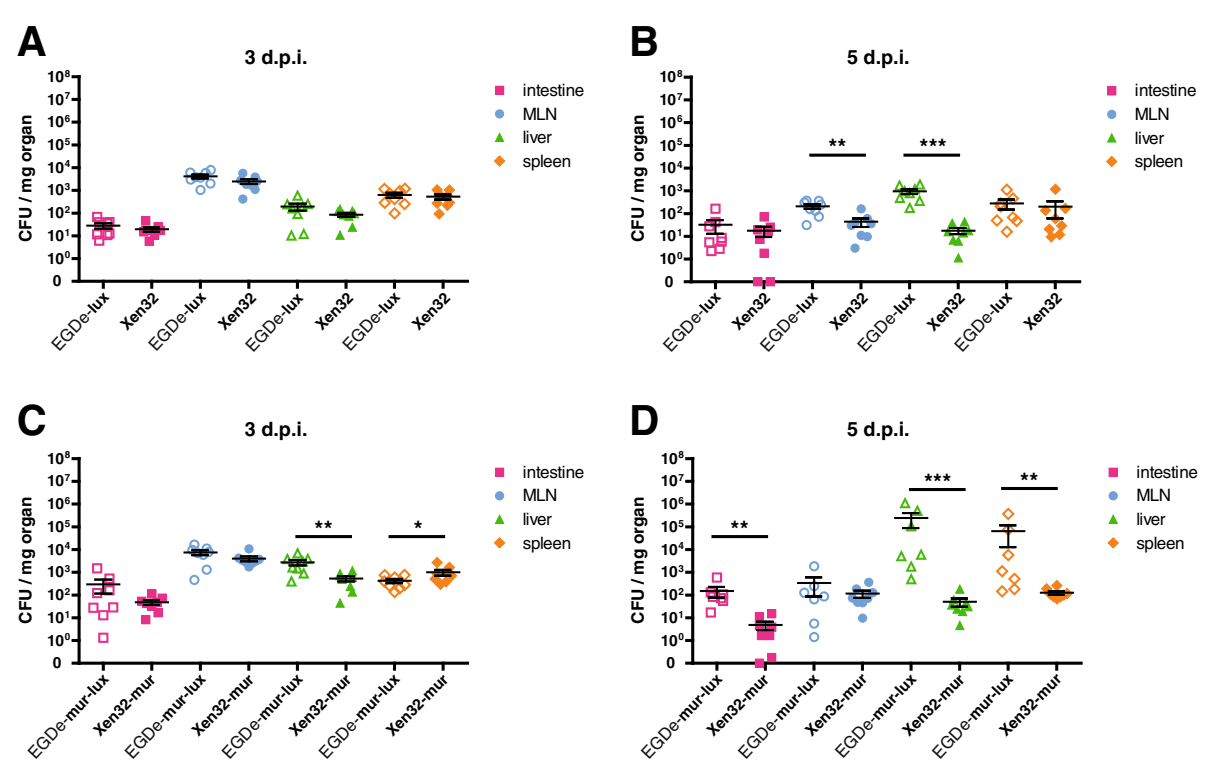

Figure 2 Lmo-Xen32 strains show reduced organ burden after oral infection of BALB/c mice compared to Lmo-EGDe strains. BALB/CJ mice $(n=8)$ were infected with $5 \times 10^{9}$ CFU Lmo-Xen32, Lmo-Xen32-mur, Lmo-EGDe-lux or Lmo-EGDe-mur-lux. At indicated time points bacterial organ loads were determined in the small intestine, mesenteric lymph nodes, liver and spleen $(n=8) .3$ d.p.i (A, C) and 5 d.p.i. (B, D) indicate 3 or 5 days post infection, respectively. Graphs demonstrated mean with standard error of bacterial load in inner organs. Statistical significance between Xen32 and EGDe strains are indicated $\left({ }^{*} p<0.05,{ }^{* *} p<0.01,{ }^{* * *} p<0.001\right.$, non-parametric Mann-Whitney-U-test).

flagellin by TLR5 and inflammasome receptors [16-19]. Previous studies have reported different effects of flagella deficiency on the pathogenesis of oral L. monocytogenes infections in vivo. The virulence of flagellin deletion mutants or mutants with deficiency in flagella regulatory proteins was found to be either increased or decreased depending on the listerial strain that was used for genetic modification or the mouse infection model that was employed $[15,17,18,20,21]$. However, these studies have used L. monocytogenes strains for oral infection
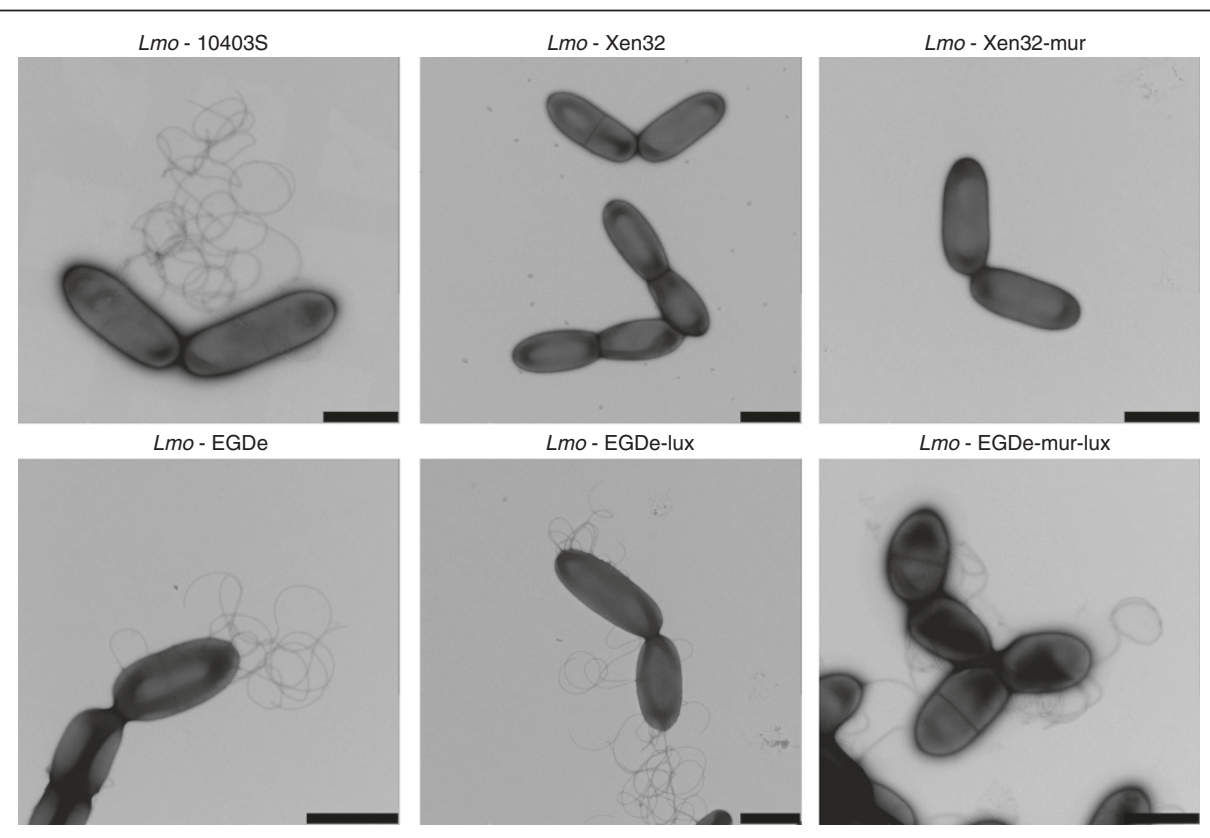

scale bar $=1 \mu \mathrm{m}$

Figure 3 L. monocytogenes Xen32 strains are flagella deficient. Transmission electron microscopy of Lmo-Xen32, Lmo-Xen32-mur, Lmo10403S, Lmo-EGDe-lux, Lmo-EGDe-mur-lux and Lmo-EGDe. Scale bars indicate $1 \mu \mathrm{m}$. 
challenge that were not optimized for InlA-mediated recognition of the murine E-cadherin receptor. We report here that Lmo-EGDe-lux shows increased in vivo virulence after oral infection challenge when compared to flagella deficient Lmo-Xen32 and that this difference in virulence between EGDe and Xen32 strains becomes even bigger when both strains are murinised for InlA.

\section{Conclusions}

The bioluminescent L. monocytogenes strain Xen32 is deficient in flagella expression and highly attenuated in virulence in an oral mouse infection model. Despite this attenuation in virulence, the L. monocytogenes strain Xen32 might be still a useful tool for in vivo imaging in experiments where sublethal or very mild infections are required (e.g. for example for phenotyping of highly susceptible or immunocompromised mouse strains). However, the scientific community should be aware that infections with $L$. monocytogenes Xen32 of wild type, immunocompetent mouse strains might result in smaller effects on host responses due to its in vivo virulence attenuation.

\section{Additional files}

Additional file 1: Figure S1. In vitro growth and luminescence profiles of Lmo-Xen32, Lmo-Xen32-mur, Lmo-EGDe-lux and Lmo-EGDe-mur-lux. Lmo-Xen32, Lmo-Xen32-mur, Lmo-EGDe-lux and Lmo-EGDe-mur-lux were grown in triplicates in $\mathrm{BH}$ media and their growth curves and emitted levels of luminescence measured as described in Material and Methods. No major differences were detected in strain growth rates but Xen32 strains emitted higher levels of luminescence at indicated timepoints.

Additional file 2: Figure S2. Bioluminescence Imaging of orally infected mice with $1 \times 10^{10}$ CFU Lmo-Xen32, Lmo-Xen32-mur, Lmo-EGDe -lux and Lmo-EGDe-mur-lux. BALB/CJ mice were intragastrically infected with $1 \times 10^{10}$ CFU Lmo-Xen32, Lmo-Xen32-mur, Lmo-EGDe-lux or LmoEGDe-mur-lux and the progress of infection was assessed by BLI for 9 days as described in Methods. Serial BLI data are shown for a set of 5 representative mice out of 10 for a time period of 9 days p.i.. Empty spaces indicate mice that succumbed to the infection or were euthanized for ethical reasons. The colour bar indicates photon emission with 3 or 4 min integration time in photons $/ \mathrm{s} / \mathrm{cm}^{2} / \mathrm{sr}$.

Additional file 3: Figure S3. Sequence of the lux-kan transposon integration site in the flaA locus of Listeria monocytogenes strain Xen32. Shown are the nucleotide and translated protein sequences. The insertion of the lux-kan transposon cassette results in a frameshift mutation with the generation of an amber stop codon (TAG) after $80 \mathrm{bp}$. Translated protein sequences of flaA are shown in blue, the frameshift protein translation is shown in red. The amber stop codon is underlined and depicted in bold red.

Additional file 4: Figure S4. Invasion and intracellular growth of LmoXen32, Lmo-Xen32-mur, Lmo-EGDe-lux and Lmo-EGDe-mur-lux. Confluent layers of Caco2 and CT26 cells were infected for 60 min with Lmo-Xen32, Lmo-Xen32-mur, Lmo-EGDe-lux and Lmo-EGDe-mur-lux. Extracellular bacteria were killed by gentamycin treatment $(100 \mathrm{\mu g} / \mathrm{ml})$. At indicated timepoints cells were washed with PBS and lysed with sterile water containing 0,2\% Triton X-100. Intracellular bacteria were quantified by plating serial dilutions of cell lysates on BHI agar plates. Graphs demonstrated mean CFU values of triplicate growth assays for each strain with standard error. Statistical significance between strains is indicated $\left({ }^{*} p<0.05,{ }^{* * *} p<0.001\right.$, non-parametric Mann-Whitney-U-test).

\section{Competing interests}

The authors declare that they have no competing interests.

\section{Authors' contributions}

SB conducted all mouse infection experiments. MR did the transmission electron microscopy analysis of the different $L$. monocytogenes strains. KS contributed to the study design and coordination of experiments. AL designed experiments, analysed data and drafted the manuscript. All authors read and approved the final manuscript.

\section{Acknowledgements}

This study was supported by grants from the National German Genome Network (NGFN-Plus, grant number 01GS0855) by the European Commission under the EUMODIC project (Framework Programme 6: LSHG-CT-2006037188) and the European COST action 'SYSGENET' (BM901), and Institute Strategic Grant funding from the BBSRC and the Helmholtz Centre for Infection Research (HZI). We thank Cormac Gahan (University College Cork, Ireland) for providing us the Lmo-EGDe-lux and Lmo-EGDe-mur-lux strains.

\section{Author details}

'Department of Infection Genetics, Helmholtz Centre for Infection Research \& University of Veterinary Medicine Hannover, Braunschweig D-38124, Germany. ${ }^{2}$ Department of Medical Microbiology, Helmholtz Centre for Infection Research, Braunschweig D-38124, Germany. ${ }^{3}$ University of Tennessee Health Science Center, Memphis, TN, USA. Infection and Immunity Division, The Roslin Institute and R(D)SVS, University of Edinburgh, Easter Bush Veterinary Campus, Edinburgh EH25 9RG, UK.

Received: 1 May 2013 Accepted: 28 June 2013

Published: 15 July 2013

\section{References}

1. Hardy J, Francis KP, DeBoer M, Chu P, Gibbs K, Contag CH: Extracellular replication of Listeria monocytogenes in the murine gall bladder. Science 2004, 303:851-853.

2. Hardy J, Margolis JJ, Contag CH: Induced biliary excretion of Listeria monocytogenes. Infect Immun 2006, 74:1819-1827.

3. Contag PR: Bioluminescence imaging to evaluate infections and host response in vivo. Meth Mol Biol 2008, 415:101-118.

4. Brandl K, Plitas G, Schnabl B, DeMatteo RP, Pamer EG: MyD88-mediated signals induce the bactericidal lectin Reglll gamma and protect mice against intestinal Listeria monocytogenes infection. J Exp Med 2007, 204:1891-1900.

5. Hardy J, Chu P, Contag CH: Foci of Listeria monocytogenes persist in the bone marrow. Dis Model Mech 2009, 2:39-46.

6. Hardy J, Kirkendoll B, Zhao H, Pisani L, Luong R, Switzer A, McConnell MV, Contag CH: Infection of pregnant mice with Listeria monocytogenes induces fetal bradycardia. Pediatr Res 2012, 71:539-545.

7. Poulsen KP, Faith NG, Steinberg H, Czuprynski CJ: Pregnancy reduces the genetic resistance of C57BL/6 mice to Listeria monocytogenes infection by intragastric inoculation. Microb Pathog 2011, 50:360-366.

8. Wollert T, Pasche B, Rochon M, Deppenmeier S, van den Heuvel J, Gruber $A D$, Heinz DW, Lengeling A, Schubert WD: Extending the host range of Listeria monocytogenes by rational protein design. Cell 2007, 129:891-902.

9. Bergmann S, Beard PM, Pasche B, Lienenklaus S, Weiss S, Gahan CG, Schughart K, Lengeling A: Influence of Internalin A murinisation on host resistance to orally acquired listeriosis in mice. BMC Microbiol 2013, 13:90.

10. Monk IR, Casey PG, Hill C, Gahan CG: Directed evolution and targeted mutagenesis to murinize Listeria monocytogenes internalin A for enhanced infectivity in the murine oral infection model. BMC Microbiol 2010, 10:318.

11. Roberts AJ, Williams SK, Wiedmann M, Nightingale KK: Some Listeria monocytogenes outbreak strains demonstrate significantly reduced invasion, inlA transcript levels, and swarming motility in vitro. App/ Environ Microbiol 2009, 75:5647-5658.

12. Mackaness GB: The Immunological Basis of Acquired Cellular Resistance. J Exp Med 1964, 120:105-120. 
13. Portnoy DA, Jacks PS, Hinrichs DJ: Role of hemolysin for the intracellular growth of Listeria monocytogenes. J Exp Med 1988, 167:1459-1471.

14. Busch DH, Vijh S, Pamer EG: Animal model for infection with Listeria monocytogenes. In Current protocols in immunology. Edited by Coligan JE. Hoboken, USA: John Wiley \& Sons, Inc; 2001. doi:10.1002/0471142735. im1909s36. Chapter 19: Unit 19.9.

15. O'Neil HS, Marquis H: Listeria monocytogenes flagella are used for motility, not as adhesins, to increase host cell invasion. Infect Immun 2006, 74:6675-6681

16. Hayashi F, Smith KD, Ozinsky A, Hawn TR, Yi EC, Goodlett DR, Eng JK, Akira $\mathrm{S}$, Underhill DM, Aderem A: The innate immune response to bacterial flagellin is mediated by Toll-like receptor 5. Nature 2001, 410:1099-1103.

17. Dons $L$, Eriksson E, Jin Y, Rottenberg ME, Kristensson $K$, Larsen CN, Bresciani J, Olsen JE: Role of flagellin and the two-component CheA/CheY system of Listeria monocytogenes in host cell invasion and virulence. Infect Immun 2004, 72:3237-3244.

18. Bigot A, Pagniez H, Botton E, Frehel C, Dubail I, Jacquet C, Charbit A, Raynaud C: Role of Flif and Flil of Listeria monocytogenes in flagellar assembly and pathogenicity. Infect Immun 2005, 73:5530-5539.

19. Zhao Y, Yang J, Shi J, Gong YN, Lu Q, Xu H, Liu L, Shao F: The NLRC4 inflammasome receptors for bacterial flagellin and type III secretion apparatus. Nature 2011, 477:596-600.

20. Grundling A, Burrack LS, Bouwer HG, Higgins DE: Listeria monocytogenes regulates flagellar motility gene expression through MogR, a transcriptional repressor required for virulence. Proc Natl Acad Sci USA 2004, 101:12318-12323.

21. Way SS, Thompson LJ, Lopes JE, Hajjar AM, Kollmann TR, Freitag NE, Wilson CB: Characterization of flagellin expression and its role in Listeria monocytogenes infection and immunity. Cell Microbiol 2004, 6:235-242.

doi:10.1186/1757-4749-5-19

Cite this article as: Bergmann et al:: The bioluminescent Listeria monocytogenes strain Xen32 is defective in flagella expression and highly attenuated in orally infected BALB/CJ mice. Gut Pathogens 2013 5:19.

\section{Submit your next manuscript to BioMed Central and take full advantage of:}

- Convenient online submission

- Thorough peer review

- No space constraints or color figure charges

- Immediate publication on acceptance

- Inclusion in PubMed, CAS, Scopus and Google Scholar

- Research which is freely available for redistribution 